\title{
Scar Symptoms: Pigmentation Disorders
}

A. Pijpe, K. L. M. Gardien, R. E. van Meijeren-Hoogendoorn, E. Middelkoop, and Paul P. M. van Zuijlen

\section{Contents}

12.1 Pathophysiology and Epidemiology - 110

12.1.1 Hypopigmentation - 110

12.1.2 Hyperpigmentation - 110

12.1.3 Maturation - 111

12.2 Measurement Techniques - 111

12.2.1 Objective Measurement Instruments -112

12.2.2 Scar Assessment Scales - 112

12.3 Therapies $\mathbf{- 1 1 2}$

12.3.1 Hypopigmentation -112

12.3.2 Hyperpigmentation -114

12.4 Conclusion -115

References - 115 


\section{Background}

To a significant degree, vascularization and pigmentation determine the color of scars. Pigmentation problems are often even more persistent than deviations in vascularization. Pigmentation problems are a common consequence after partial and full-thickness burns, skin pathology such as vitiligo, other skin trauma, and surgical procedures. Almost all these patients experience at least some pigmentation problems. Both hypopigmentation and hyperpigmentation can cause esthetic and psychological issues which affect the quality of life of patients. Information on the role and pathways of melanocytes in pigmentation problems and the changes that occur during scar maturation is increasingly more understood. Different subjective and objective methods are available to determine scar color. Hypo- and hyperpigmentation management options include surgical and nonsurgical techniques.

\subsection{Pathophysiology and Epidemiology}

Altered skin pigmentation, or pigmentation disorders, can result from increased or decreased melanin, abnormal melanin distribution, decreased hemoglobin, or deposition of exogenous substances. Melanin is produced by melanocytes, specialized cells of neural crest origin that reside in the basal layer of the epidermis. The biosynthesis of melanin occurs in lysosome-like organelles called melanosomes, which are transported to the cell periphery and transferred from the dendritic tips of the melanocytes to the surrounding keratinocytes. Each melanocyte is associated with approximately 36 basal keratinocytes to form the so-called "epidermal melanin unit."

\subsubsection{Hypopigmentation}

Hypopigmentation is characterized by skin becoming lighter than the individuals own color, but not completely lacking pigment (• Fig. 12.1). This should not be confused with depigmentation, which is the absence of all pigment. Hypopigmentation is common and approximately 1 in 20 have at least one hypopigmented macule [1]. Common causes include vitiligo, postinflammatory hypopigmentation, pityriasis versicolor, pityriasis alba, and halo naevi. Hypopigmentation disorders may be congenital or acquired. Acquired hypopigmentation may be the result of inflammatory conditions or trauma like a burn injury. In burn scars, hypopigmentation is a frequent pathology. Although the condition often improves over time with scar maturation, lasting depigmented or hypopigmented lesions may remain.

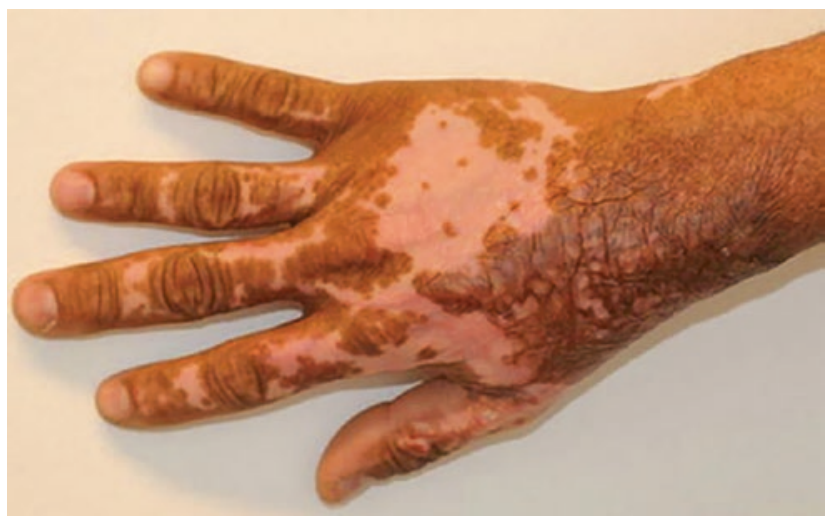

Fig. 12.1 Hypopigmentation in a hand after burn injury

Data on the prevalence of these conditions after burn injury are scarce.

Vitiligo is an autoimmune disease where melanocytes are affected. The condition affects approximately $0.5-1 \%$ of the population, occurs in patients of all ages, equally affects men and women, with an early onset in many cases $(<20$ years). There is no difference in prevalence with respect to skin type or race.

\subsubsection{Hyperpigmentation}

Hyperpigmentation is the darkening or increase in the natural color of the skin and results from overproduction or abnormal release of melanin in the epidermis and/or dermis in response to either endogenous or exogenous inflammatory conditions [2]. Superficial hyperpigmentation is located in the epidermis and causes a light- to dark-brown discoloration. As soon as the pigment lies deeper in the skin (dermal pigmentation), the hyperpigmentation acquires a gray-brown to gray-blue glow. Like hypopigmentation, hyperpigmentation disorders may be congenital or acquired.

Postinflammatory hyperpigmentation is a reactive hypermelanosis of the skin that occurs as a sequela of cutaneous inflammation. Common causes of include acne vulgaris, eczematous dermatoses, and burn injury. Postinflammatory hyperpigmentation may also occur as a complication of a chemical peeling.

Although this pigmentary change can be observed in all skin types, it more frequently affects individuals with higher degrees of skin pigmentation (meaning Fitzpatrick skin types IV-VI) due to increased reactivity of melanocytes within the skin. The modified Fitzpatrick skin type classification system contains six categories with types I-IV describing different types of white skin and type V "brown" and type VI "black" skin.

Severe burns significantly affect the process of pigmentation as it is tightly regulated by cell proliferation 


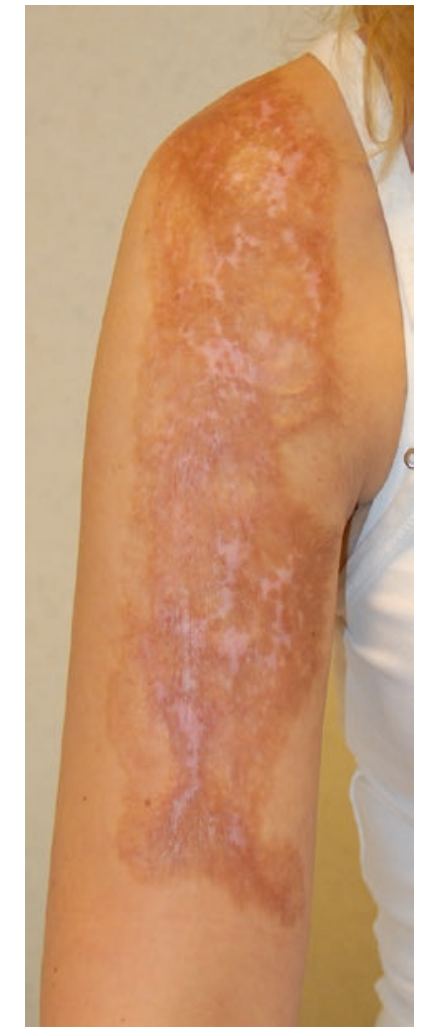

- Fig. 12.2 Burn scar with hyperpigmented and hypopigmented areas

and differentiation of melanocytes and melanocyte stem cells which are located in the epidermis and hair follicles of the skin. Almost all burn patients with deep partialthickness wounds and full-thickness wounds experience at least some pigmentation problems (• Fig. 12.2). Superficial burn wounds generally heal without pigmentation problems. Although scar-related hypo- and hyperpigmentation are not harmful, it can cause significant cosmetic problems and become a great psychological burden for patients which influences their quality of life.

\subsubsection{Maturation}

Maturation is the fourth and final stage of the wound healing process and is commonly referred to as remodeling. The cellular changes that occur during scar maturation are increasingly understood. They are associated with remodeling of the extracellular matrix as well as normalization of the ratio of type III to type I collagen. However, the process of scar maturation with regard to changes in clinical appearance over time is the least understood part of wound healing. Most studies suggest a mean maturation period of 1,2 , or several years. In this active stage it can be difficult to determine to what extent deviant skin color is caused by vascularization and/or pigmentation. By pressing the scar with a finger
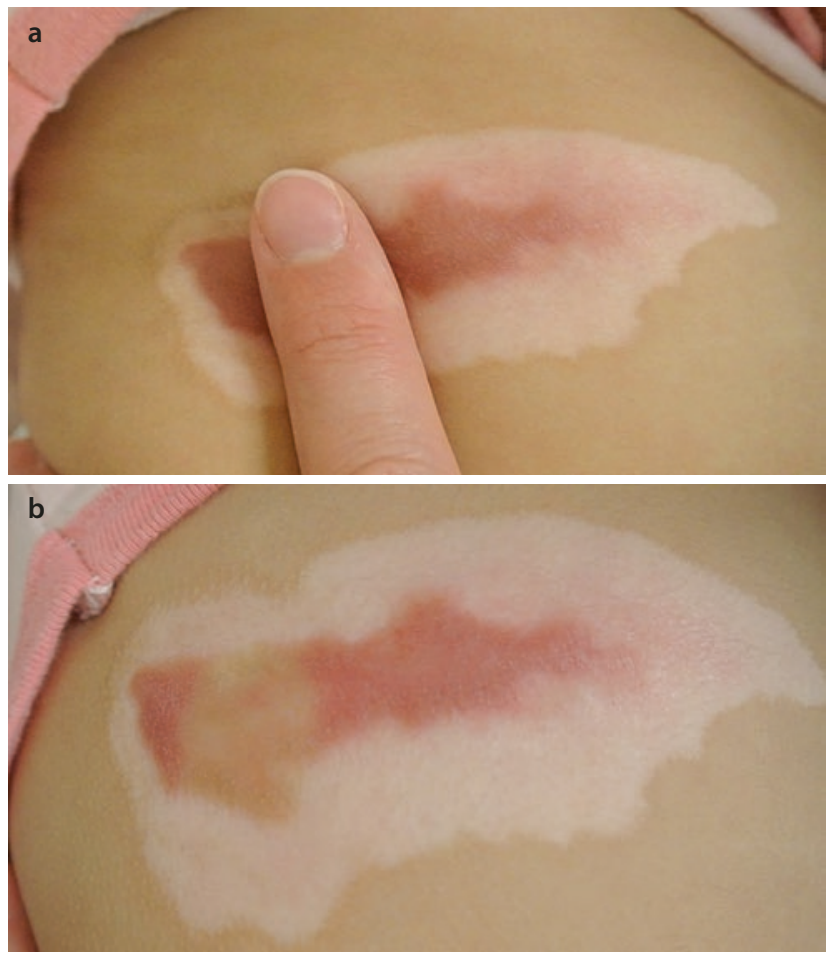

- Fig. 12.3 (a) Pressure on the scar with a finger reduces blood flow in the scar. (b) Immediately after release of the pressure, level of pigmentation of the scar is visible

or transparent ruler like made from plexiglass, the blood flow is reduced and degree of pigmentation can be better established (• Fig. 12.3).

Van der Wal et al. performed the first longitudinal study on the maturation pattern of individual scar characteristics and predictors of severe scarring in a representative burn population [3]. They conducted a detailed analysis on the clinical changes of burn scars in 474 patients following a longitudinal scar assessment protocol. The observations of the POSAS Observer Scale (see - Sect. 2.2) showed that all scar parameters, except vascularization, become more apparent in the first 6 months after the injury. After 12 months, mean POSAS scores were significantly lower for vascularization, pigmentation, and pliability compared with the 6-month followup. Statistical significant improvement at 12 months was also found for the parameters vascularization and pigmentation compared with the 3-month evaluation.

\subsection{Measurement Techniques}

For the diagnosis, evaluation of progress of disease and/or therapy it is of major importance to be able to measure skin or scar color in a noninvasive, valid, and reliable way. Outcome measures receive more and more attention to evaluate therapies on an individual basis as 
well as at group level for efficacy studies. For this, validated techniques are a prerequisite.

To determine scar color, different subjective and objective methods are developed and available.

\subsubsection{Objective Measurement Instruments}

Several instruments are available to measure skin or scar color. The output is generally a combination of redness (erythema) and melanin (pigment). Most instruments use either narrow band spectrophotometry or reflectance colorimetry. The tristimulus reflectance colorimetry measures color through three broad wavelengths filters representing brightness, redness, and pigmentation. This system is suitable to measure any kind of color in the standard color space defined by Commission Internationale de l'Eclairage (CIE), where the output in $\mathrm{L} * \mathrm{a} * \mathrm{~b}$ coordinates is defined as CIELAB color space values [4]. The $\mathrm{LAB}$ values express color as three values: $\mathrm{L} *$ for the lightness (black to white), $\mathrm{a} *$ from green to red, and $\mathrm{b} *$ from blue to yellow.

Narrow-band reflectance spectrophotometers measure erythema and melanin index, representing vascularization and pigmentation, based on differences in light absorption by hemoglobin and melanocytes, respectively, in selected bands of the light spectrum corresponding to hemoglobin and melanin absorption spectra.

Van der Wal et al. performed a study where the reliability, validity, and feasibility of narrow-band reflectance spectrophotometry and tristimulus reflectance colorimetry on normal skin and scar tissue were studied [5]. All three devices included in this study, Mexameter (narrow band spectrophotometer) and Colorimeter (tristimulus reflectance) (both from Courage \& Khazaka Electronic GmbH, Cologne, Germany) and DSM II ColorMeter (narrow band spectrophotometer) (Cortex Technology, Hadsund, Denmark), obtained reliable results after a single measurement, performed by one observer.

Some aspects of these measurements need to be considered when using skin color data for therapeutic evaluation: Skin color is generally adapted to seasonal differences and is likely to be different on various anatomical regions of the body. Therefore, measurements of skin or scar lesions on specific locations need to be compared to the relevant normal skin of a nearby or contralateral comparative anatomical region. For narrow band spectrophotometric measurements, this needs to be performed by subtraction of the data of the normal skin from the lesional data. Furthermore, for the narrow band spectrophotometers, some caution is needed in interpretation of the data from highly pigmented skin: There is some influence on the erythema index by the melanin index, in that the erythema index apparently increases with increasing melanin index. Also, the melanin index is influenced by the oxygen saturation level of hemoglobin, since reduced hemoglobin absorbs more red light than oxyhemoglobin. For example, lowering the arm of a subject to be measured will lower the oxygenation, and give an increase in both erythema and melanin indices. Therefore, a standardized position for the measurements is recommended.

\subsubsection{Scar Assessment Scales}

Today, many scales exist to evaluate skin and scar characteristics [6]. One of the earliest and frequently reported is the Vancouver Scar Scale (VSS) [7]. Despite the fact that it is frequently used as a scale, especially the items "erythema" and "pigmentation" are nominal categories rather than ordinal ones. For the item "pigmentation" categories are normal, hypo, mixed, or hyper pigmentation; and for the item 'erythema' they are normal, pink, red, and purple. The VSS gives no intensity value of these parameters and therefore the numbers that are often attributed to these categories cannot be added to give a "severity score" for total scar characteristics. This is specifically important for pigmentation problems, since "hypopigmentation" cannot generally be considered to be less severe than "hyperpigmentation," which would be a consequence if the VSS categories would be used as a true scale.

To overcome these difficulties, the Patient and Observer Scar Assessment Scale (POSAS) was designed and validated [8]. The POSAS questionnaire represent the severity of burn scar quality including parameters that indicate skin color and pigmentation on a numerical 10-point rating scale. Furthermore, the categories "pale, pink, red, purple, mix" and "hypo, hyper, mix" are added for each assessment. Within these categories, the numerical data can be added to present an overall scar severity score.

\subsection{Therapies}

\subsubsection{Hypopigmentation}

\subsubsection{Nonsurgical Techniques}

\section{Laser Therapy}

Laser therapy can be used to treat pigment disorders in a scar. If there is hypopigmentation of a scar, it can be treated with the Excimer laser (308 nm) [9]. This laser produces a stimulation of melanocytes, thereby correcting hypopigmentation in the scar tissue. 
Other pigment lasers mainly focus on the presence of hyperpigmentation and induce pigment reduction.

\section{Dermatography}

Dermatography, also known as medical tattooing, is an alternative method to improve the scar color, particularly in hypopigmented areas. Chemical-based pigments are injected into the dermis, as with other sorts of tattooing. Dermatography can be repeated if the color fades over time.

\section{Camouflage Therapy}

Camouflage therapy offers a temporary solution to hide a (hypopigmented) scar or make it less visible. Often a cream, spray, or powder that is close to the original skin color is used. Once the correct color has been determined, the application can be used independently in the home situation.

\subsubsection{Surgical Techniques \\ Dermabrasion}

Dermabrasion can help to treat the hypopigmentation of a scar.

By abrasion, the epidermis and a very small layer of the dermis are removed layer by layer, resulting in a superficial wound.

Normally, the abrasia is done until the whitish dermal layer is exposed with no more than fractional bleeding present.

Dermabrasion is performed as a single treatment modality or in combination with other treatments such as skin transplantation or cell spray. If it is used as a single treatment modality, the superficial wound has to recover by itself. In those cases abrasia must be carried out carefully to prevent wound-healing problems if the wound becomes too deep. This method is mainly used in case of hyperpigmentation where the excess pigment is shaved off and the new epidermis hopefully has a more balanced pigment and therefore color (see below).

In hypopigmentation the dermabrasion is mostly combined with a method to transplant melanocytes from healthy skin to the depigmented regions like skin grafting or cell therapy.

\section{Skin Grafting}

Skin-grafting techniques include split-thickness skin grafts, full-thickness skin grafts, punch-grafting, and epidermal grafts, such as grafts from suction blisters.

The split skin graft (SSG) contains the entire epidermis with only a thin layer of dermis. The skin is harvested with a dermatome preferably from a suitable, color-matching concealed donor site. A pigmented donor area with a thickness of $0.15 \mathrm{~mm}$ is identified to achieve optimal outcomes as the basal layer of the epidermis and a complement of melanocytes remain intact.

The full-thickness graft provides better functional and cosmetic outcomes in terms of scarring compared to the SSG. The entire epidermis and dermis are transplanted. For correction of pigment disorders, this is only indicated if the general quality of the scar is unacceptable/problematic and the scar area is limited.

In skin grafting, selecting a donor site of healthy skin with suitable normal pigmentation is key to ensure optimal outcomes.

Small full-thickness punch biopsies are harvested from healthy skin from a concealed body part, normally the upper arm or upper leg. The size of the biopsies can be $1-4 \mathrm{~mm}$ in diameter. Repigmentation occurs in $70 \%$ of the cases. Melanocytes migrate from the punch biopsies into the adjacent area in a centrifugal manner. A disadvantage of punch grafting is the cobblestone appearance that may remain in the long term.

Grafting of suction blisters is an interesting and successful technique for the treatment of hypopigmentation disorders. Negative pressure is exerted on healthy skin of the patient to create epidermal blisters. Devices such as a suction pump, vacuum bottle, hoses, or suction cups can be used for harvesting. This blister is harvested subsequently and transplanted to the depigmented area.

The donor site generally heals spontaneously with no or minimal scarring. Minor adverse effects such as donor site hyperpigmentation and color mismatching may be encountered.

\section{Cell Therapy}

An autologous epidermal cell suspension spray can be used to enhance repigmentation. The suspension can be sprayed on top of the wound after dermabrasion or injected in the hypopigmented area. This was studied by Falabella et al. already in 1971 and measured both by clinical score and by melanocyte counts, but the difference between the groups was not statistically significant in either case [10].

Later the RECELL ${ }^{\circledR}$ Autologous Cell Harvesting Device (AVITA Medical Europe Ltd., Melbourne, UK) became available, which uses an on-site device to isolate epidermal cells from a small piece of split skin graft.

Similar to the treatment of vitiligo, melanocytes are directly isolated and harvested from skin grafts prior to transplantation to the depigmented injured area. These cell-based treatments can be divided into (i) non-cultured cell suspension, (ii) cultured melanocyte suspension, (iii) cultured keratinocyte/melanocyte suspension, (iv) 
tissue-engineered melanocyte grafts. For current clinical practice mainly the non-cultured cell suspension is used. The other, more sophisticated options may be interesting but are rarely used as clinical therapy so far. These therapies are expensive and laborious and currently it has not been conclusively demonstrated that they are cost effective.

A limitation of cell transplantation is a potentially uneven cell dispersion across the recipient site. Furthermore, poor attachment of transplanted melanocytes to the recipient skin and unfavorable wound conditions may also affect cell survival and outcome.

\section{Microneedling}

Microneedling is a relatively new therapeutic modality in dermatology. It has shown promising results as an adjuvant therapy for enhanced drug delivery in the treatment of atrophic scars, alopecia, actinic keratoses, and disorders of pigmentation such as melisma [11]. The efficacy in treatment of vitiligo remains limited.

\section{Excision}

Excision of the hypopigmented area can be performed and needs to be combined with a technique for wound closure. For small areas direct wound closure is the first choice. For larger areas and in the case of considerable tension on the wound edges, additional closing techniques such as the skin stretching technique or tissue expansion can be performed.

The skin stretch technique is suitable for closing defects after excision of scars and wounds of limited size. The skin stretcher is a device that can stretch two widely spaced wound edges together. The force with which this goes can be set, but is usually in the order of 30 Newton. The best result is obtained when working in cycles of 4 minutes of stretch and 1 minute of rest. After a few cycles, the wound tension has dropped and the wound can be closed under acceptable tension. In a large study, the number of wound dehiscence was limited [12].

With the help of a tissue expander large areas of scar can be normally excised. The tissue expander technique is widespread and is frequently used for reconstruction of large scar areas such as alopecia areas of the hairy head. During the first operation, an expander is placed under flexible skin in the area of the scar area that needs to be reconstructed. This extension is a special balloon with a filling port. The filling port is also placed under the skin. The fill port can be triggered through the skin so the balloon can be filled in tempi until sufficient texture has been expanded for the scar reconstruction. However, despite good results, two interventions are needed, the total procedure may take several weeks to months with frequent visits to the clinic to fill the balloon.

\subsubsection{Hyperpigmentation}

\subsubsection{Nonsurgical Treatment Topical Treatments}

Hydroquinone, either as monotherapy or in combination with alpha-hydroxy acid, ascorbic acid, retinoids, corticosteroids, and antioxidants, is a commonly used depigmenting agent [2]. Its working mechanism is thought to inhibit melanogenesis by acting as an alternative substrate for the enzyme tyrosinase. Hydroquinone may also result in inhibition of DNA/RNA synthesis, destruction of melanocytes, and degradation of melanosomes.

Topical retinoids, including tretinoin (all-transretinoic acid), tazarotene, and adapalene, are also used as monotherapy for treatment of hyperpigmented lesions. Retinoids are supposed to act by inhibition of tyrosinase, induction of melanocyte apoptosis, and acceleration of epidermal cell turnover.

Also azelaic acid is thought to inhibit tyrosinase and decrease melanogenesis.

\section{Chemical Peels}

A commonly used acid against hyperpigmentation is the use of lactic acid. Lactic acid is particularly known to prevent the formation of tyrosinase so that pigment cells can form less pigment. In addition, the accelerated exfoliation of the skin cells fades the pigment cells present. The mechanism of this effect might be due to epidermal remodeling and accelerated desquamation, which would result in quick pigment dispersion.

\section{Laser Therapy}

Hyperpigmentation can be treated with laser therapy, based on selective photothermolysis. Selecting the right wavelength can tackle pigment selectively. Selective photothermolysis suggests that laser therapy would allow discriminating destruction of pigment without injuring the surrounding tissue. Selective melanin photothermolysis can be obtained with any laser light having a wavelength in the absorption spectrum of melanin and sufficient energy levels to target melanosomes (mostly used: the Q-switched ruby laser (694 nm), Q-switched $\mathrm{Nd}$ :YAG laser $(532 \mathrm{~nm}, 1064 \mathrm{~nm})$, and the Q-switched alexandrite laser $(755 \mathrm{~nm})$ ). Laser induces extreme heating of melanosomes with subsequent thermal expansion, local vaporization and generation of acoustic waves that damage the nucleus and eventually destroy the pigment-laden cells. The released melanin is then removed through transepidermal elimination or phagocytosis by dermal macrophages. To be effective and specific, wavelengths that avoid absorption by other skin chromophores and penetrate to the desired depth have to be used [13]. 


\subsubsection{Surgical Treatment Dermabrasion}

As mentioned above, dermabrasion can help to reduce hypo- and hyperpigmentation of a scar. In the case of hyperpigmentation, dermabrasion is usually performed as a single treatment modality, that is, it is not combined with another treatment such as skin transplantation. The idea is to easily remove the epidermis including the melanocytes and the pigment and let it heal spontaneously resulting in an epidermis with a lower concentration of melanocytes. It is not recommended to go too deep because the regeneration capacity of the skin is limited in scars, due to lowered presence or even absence of hair follicles and sweat glands. If the wounds are too deep and stay open too long, problematic new scars can occur.

It is advisable to perform dermabrasion in the fall or winter season to anticipate low UV light exposure to reduce the risk of excessive new pigmentation. It is also recommended to start dermabrasion with a small test area, preferably in an area that can be easily evaluated by the patient.

\section{Excision}

Excision and closure is also a feasible therapeutic option for hyperpigmented lesions that are limited in size (see above under hypopigmentation). It should be taken into account that a (linear) scar will remain and that outcome may be more severe than the original situation.

\subsection{Conclusion}

Pigmentation problems are an important feature of scar quality and partly determine the visual characteristics of a scar. Both hypopigmentation and hyperpigmentation can cause aesthetic and psychological issues which influences quality of life. Pigmentation disorders are difficult to treat. Treatment options include camouflage therapy, topical treatments, chemical peels, laser therapy, dermatography, dermabrasion, microneedling, skin grafting, cell therapy, and excision. It is advised to first try the conservative options and to dose the treatment to prevent overshoot and complications.
Take-Home Messages

- Pigment abnormalities are divided into hyperpigmentation and hypopigmentation.

- Pigmentation disorders are difficult to treat.

- Nonsurgical and surgical options are available.

- Wait until the area has matured.

- First try the conservative options. If possible, first test the response to the therapy in a test area.

- Dose the treatment to prevent overshoot and complications.

\section{References}

1. Hill JP, Batchelor JM. An approach to hypopigmentation. BMJ. 2017;356:i6534.

2. Kaufman BP, Aman T, Alexis AF. Postinflammatory hyperpigmentation: epidemiology, clinical presentation, pathogenesis and treatment. Am J Clin Dermatol. 2018;19(4):489-503.

3. van der Wal MB, et al. Outcome after burns: an observational study on burn scar maturation and predictors for severe scarring. Wound Repair Regen. 2012;20(5):676-87.

4. Takiwaki H. Measurement of skin color: practical application and theoretical considerations. J Med Invest. 1998;44(3-4):121-6.

5. van der Wal M, et al. Objective color measurements: clinimetric performance of three devices on normal skin and scar tissue. $\mathrm{J}$ Burn Care Res. 2013;34(3):e187-94.

6. van der Wal MB, et al. A clinimetric overview of scar assessment scales. J Burn Care Res. 2012;33(2):e79-87.

7. Sullivan T, et al. Rating the burn scar. J Burn Care Rehabil. 1990;11(3):256-60.

8. Draaijers LJ, et al. The patient and observer scar assessment scale: a reliable and feasible tool for scar evaluation. Plast Reconstr Surg. 2004;113(7):1960-5; discussion 1966-7.

9. Alexiades-Armenakas MR, et al. The safety and efficacy of the 308-nm excimer laser for pigment correction of hypopigmented scars and striae alba. Arch Dermatol. 2004;140(8):955-60.

10. Falabella R. Epidermal grafting. An original technique and its application in achromic and granulating areas. Arch Dermatol. 1971;104(6):592-600.

11. Iriarte $\mathrm{C}$, et al. Review of applications of microneedling in dermatology. Clin Cosmet Investig Dermatol. 2017;10:289-98.

12. Verhaegen PD, et al. Sustainable effect of skin stretching for burn scar excision: long-term results of a multicenter randomized controlled trial. Burns. 2011;37(7):1222-8.

13. Briganti S, Camera E, Picardo M. Chemical and instrumental approaches to treat hyperpigmentation. Pigment Cell Res. 2003;16(2):101-10.

\footnotetext{
Open Access This chapter is licensed under the terms of the Creative Commons Attribution 4.0 International License (http://creativecommons. org/licenses/by/4.0/), which permits use, sharing, adaptation, distribution and reproduction in any medium or format, as long as you give appropriate credit to the original author(s) and the source, provide a link to the Creative Commons license and indicate if changes were made.

The images or other third party material in this chapter are included in the chapter's Creative Commons license, unless indicated otherwise in a credit line to the material. If material is not included in the chapter's Creative Commons license and your intended use is not permitted by statutory regulation or exceeds the permitted use, you will need to obtain permission directly from the copyright holder.
} 